\title{
BEITRÄGE
}

\section{Kurfürst Friedrich der Weise und die Wittenberger Augustiner-Eremiten Anmerkungen zur Gründung und materiellen Ausstattung des Klosters}

von

UWE SCHIRMER

Empirisch gesättigte Aussagen zur Gründung und zur Ausstattung des Wittenberger Augustiner-Eremitenklosters sind aufgrund der schwierigen Quellenlage problematisch. Das aussagekräftigste Material wurde von Gottfried Wentz im Rahmen seiner Arbeit an der Germania Sacra zusammengetragen. ${ }^{1}$ Da jedoch die Ansiedlung der Augustiner-Eremiten im Zusammenhang mit der Gründung der Universität Wittenberg steht und es intensive Beziehungen zwischen Konvent und Leucorea gab, haben vor allem die Forschungen zur Finanzierung der Wittenberger Hohen Schule einerseits sowie die baugeschichtlichen Untersuchungen zum Kloster anderseits Fragen und Probleme aufgeworfen, die wiederum weiterführende Rückschlüsse auf die materielle Ausstattung des Augustinerklosters zulassen. ${ }^{2}$ Zudem förderte die Durchsicht der Wittenberger Amtsrechnungen sowie eines Zinsregisters von 1503 einige bisher unbekannte Details zutage.

Für die schlechte Quellensituation ist nicht zuletzt die kurze Lebensdauer des Klosters ausschlaggebend, konnte es doch nur knapp zwei Jahrzehnte mit Stiftungen und Schenkungen bedacht werden. Selbst der Baubeginn liegt im Dunkeln. 1502 soll die Grundsteinlegung erfolgt sein, freilich ist dies strittig. Gesichert ist

1 Fritz Bünger/Gottfried Wentz (Bearb.), Die Bistümer der Kirchenprovinz Magdeburg. Das Bistum Brandenburg (Germania Sacra, 3. Band, 2. Teil), Berlin 1941, S. 440499, besonders S. 453-457.

2 Reinhard Schmitt, Zur Baugeschichte des Augustiner-Eremitenklosters in Wittenberg, in: Harald Meller u. a. (Hg.), Luthers Lebenswelten (Tagungen des Landesmuseums für Vorgeschichte Halle 1), Halle/Saale 2008, S. 177-191; Gerhart Enders, Die wirtschaftliche Ausstattung der Universität Wittenberg 1502-1547. Die Entstehung der landesfürstlichen Universität, Phil.-Diss. Halle-Wittenberg 1952 (masch.), S. 11-16; Uwe Schirmer, Die finanziellen Grundlagen der Universitäten Leipzig, Wittenberg und Jena im Vergleich (1409-1633), in: Stefan Michel/Christian Speer (Hg.), Georg Rörer (1492-1557). Der Chronist der Wittenberger Reformation (Leucorea-Studien zur Geschichte der Reformation und der Lutherischen Orthodoxie 15), Leipzig 2012, S. 75-103, hier S. 86 f. 
hingegen, dass der sächsische Kurfürst am 6. Juni 1504 den Wittenberger Stadtrat anwies, den Augustinermönchen das durch den Praeceptor der Lichtenburger Antoniter abgemessene Grundstück beim einstigen Hospital zum Heiligen Geist zu übergeben, um das Kloster statlich und fürderlich erbauen zu können. ${ }^{3}$ Die Kämmereirechnung der Stadt Wittenberg bestätigt für das Rechnungsjahr 1503/04, dass spätestens 1504 die Augustiner diesen Platz eingenommen hatten. ${ }^{4}$

\section{Das Heilig-Geist-Hospital}

Im Laufe des Jahres 1504 begannen die Augustiner, die Klostergebäude eben an jener Stelle errichten zu lassen, wo das Heilig-Geist-Hospital stand und wo sich heute das Luther-Haus befindet. Dies belegt nicht zuletzt des Kurfürsten Sorge bezüglich der Bedürftigen des Heilig-Geist-Spitals, dass in der südöstlichen Ecke der Wittenberger Altstadt Baumaßnahmen anstanden. In einem an den Stadtrat gerichteten Brief des Fürsten vom 17. Juni 1504 heißt es, dass das Baugelände nunmehr übergeben und der Orden willens sei, mit dem Bau zu beginnen und etliche Brüder im neuen Kloster anzusiedeln. Um den Konvent bei seinem Vorhaben nicht zu behindern, möge der Rat dafür sorgen, dass die Spitalsinsassen eine andere Unterkunft bekämen. ${ }^{5}$ Scheinbar verlief die Verlegung des Spitals reibungslos, denn sowohl 1503/04 als auch 1504/05 wurden den armen lewtthen zcum heyligen geist und heiligen creutzen vier Scheffel Malz aus dem Amt Wittenberg gereicht. ${ }^{6}$ Diese Tatsache ist insofern bedeutsam, weil das Hospital als Rechtsform natürlich weiter existierte. Inwieweit den Augustinern zusätzlicher Besitz und weitere Einkünfte übertragen wurden, bleibt offen. An und für sich hätte, außer dem Baugrund, alles beim Hospital bleiben müssen. Allerdings verzeichnet eine Akte aus dem Jahr 1504, dass die Augustiner vom Heilig-Geist-Spital etliche (!) wohlgebaute Häuser und zwei Brauerben übereignet bekamen; zudem ein zum Heilig-Kreuz-Hospital gehörendes Haus, welches vor der Stadt lag.7 Das vor den Toren der Stadt liegende Gebäude könnte das sogenannte Haus Bruno oder auch das kleine Haus gewesen sein, welches der letzte Prior des Augustinerklosters, Eberhard Brisger, zu Beginn des Jahres 1525 vom Kurfürsten auf Luthers Bitte geschenkt bekam. ${ }^{8}$ Auf welchem Grundstück die Rechte für die beiden Gebräue hafteten, ist unbekannt. Wahrscheinlich werden sie auf einem Haus gelegen

3 BüngeR/Wentz, Bistum Brandenburg (wie Anm. 1), S. 444.

4 Bünger/Wentz, Bistum Brandenburg (wie Anm. 1), S. 444.

5 Thüringisches Hauptstaatsarchiv Weimar (im Folgenden: HStA Weimar), EGA, Reg. Kk 1425, fol. $1^{\mathrm{r}}$.

6 HStA Weimar, EGA, Reg. Bb 2742, fol. $132^{\mathrm{r}}$ (1503/04); Reg. Bb 2744, fol. $118^{\mathrm{v}}$ (1504/05).

7 Item etliche wolgebawthe heuser, item eyn haus, dem heyligen crentze vorm thor zw gehorigk, item zwey brewerbe (...). HStA Weimar, EGA, Reg. Kk 1425, fol. $4^{\mathrm{r}}$.

8 Vgl. Johann Karl Seidemann, Luthers Grundbesitz, in: Zeitschrift für die historische Theologie 30 (1860), 4, S. 475-570, hier S. $502 \mathrm{f}$. 
haben, welches als Freihaus in der Bürgermeistergasse stand. Das Erbbuch des Amtes Wittenberg von 1513 erwähnt zumindest jeweils zwei Freihäuser, die den Franziskanern und Augustinern gehörten. Das Freihaus der Augustiner-Eremiten hatte zu dieser Zeit der Amtsschösser Antonius Niemegk inne. ${ }^{9}$ Es soll das einstige Terminierhaus der Herzberger Augustiner gewesen sein, das 1504 als Eigentum an den Wittenberger Konvent übergegangen war. ${ }^{10}$ Doch wo befanden sich die „etlichen wohlgebauten Häuser“, die 1504 mit übergeben worden sind? Lagen die Häuser oder Höfe am Ende der „Rossen“-Straße vor dem Elstertor? Auch war eine alte Ziegelscheune an die Augustiner gekommen. Diese Ziegelscheune wird in einer Stadtkassenrechnung von 1522 als die der Augustiner bezeichnet, in dem Jahr wurde sie auf Kosten des Rates abgebrochen. Gottfried Wentz vermutet, dass die beiden erwähnten Höfe vor dem Elstertor, die mit Stadtsteuer belasteten Gärtnerhöfe waren, wo die Augustiner bereits 1508 im Zahlungsrückstand lagen. ${ }^{11}$

Der grundherrliche Besitz verblieb beim Heilig-Geist-Hospital. ${ }^{12}$ Zwar gelangte die Kapelle des Hospitals, die sich auf dem Gelände des Klosters befand, in Besitz des Ordens, allerdings konnte er die an ihr haftenden Rechte nicht übernehmen bzw. durchsetzen. Ausdrücklich wird dies im Erbbuch des Amtes Wittenberg von 1513 betont: Des beiligen geists capellen, dor aufs ytzt das augustinercloster gebawet, dor ynne sindt 3 lebn, eyns hat der pfarrer zu Dobyn unde zwey der rath $z u$ verleiben. ${ }^{13}$ Diese Tatsache wurde von den Visitatoren im Jahr 1528 bestätigt. Das Patronatsrecht lag beim Rat, ohne etwaiger Anrechte der Augustiner auch nur zu gedenken. ${ }^{14}$ Kurzum: Infolge der Übernahme des Grundstücks des HeiligGeist-Hospitals wurden dessen Einkünfte nur marginal geschmälert; dies betraf die beiden Brauerben. Ansonsten erlangten die Augustiner keine Ansprüche an Benefizien, die an der Kapelle des Hospitals anhängig waren. Sie übernahmen nur die baufällige Kapelle sowie das dazugehörige Inventar.

Über den schlechten Zustand der Kapelle informiert Friedrich Myconius: $Z u$ Wittenberg war das Augustinerkloster neu angefangen zu bauen und nicht mehr denn das Schlafhaus, darin jetzt Dr. Martinus noch wobnet, ausgebauet. Die Fundamenta der Kirchen waren angelegt, aber nur der Erden gleich bracht. Mitten in denselben Fundamentis stund ein alt Kapellen von Holz gebauet und mit Lebmen bekleibt, das war sehr baufällig, war gestützelt auf allen Seiten. Es war etwa, wie

9 Otto Oppermann, Das sächsische Amt Wittenberg im Anfang des 16. Jahrhunderts. Dargestellt auf Grund eines Erbbuches vom Jahre 1513, Leipzig 1897, S. 85.

10 Bünger/Wentz, Bistum Brandenburg (wie Anm. 1), S. 456.

11 Bünger/Wentz, Bistum Brandenburg (wie Anm. 1), S. 455 f.

12 Mitte des 15. Jahrhunderts gehörten sechs Hufen in der Wittenberger Flur und das Dorf Dobien mit 16 Hufen sowie mit Kirche und Pfarrlehn zum Heilig-Geist-Spital. In Dobien saßen vier Hüfner und drei Kossäten, die dem Spital grundherrlich unterstanden. BüNGER/WENTZ, Bistum Brandenburg (wie Anm. 1), S. 161.

13 Zitiert nach: Oppermann, Amt Wittenberg (wie Anm. 9), S. 111.

14 Karl Pallas (Hg.), Die Registraturen der Kirchenvisitationen im ehemaligen sächsischen Kurkreise (Geschichtsquellen der Provinz Sachsen 41, Abt. II/1), Halle/Saale 1906, S. 109. 
ich's gesehen hab, bei 30 schuhen lang und 20 breit. Hatt ein klein rostig Burkirchlein [sc. Empore], darauf ein zwanzig Menschen mit Not stehen kunnten. An der Wand gegen Mittag war ein Predigtstubl von alten Brettern, die ungebobelt: ein Predigstüblchen, gemacht, etwa anderthalb Ellen hoch über dem Erdboden. In Summa: Es hatt allenthalben das Ansehen, wie die Maler den Stall malen zu Bethlehem, darin Christus geboren war. ${ }^{15}$ Mit der Kapelle waren auch liturgische und gottesdienstliche Utensilien dem Kloster übergeben worden. Das aus dem Jahr 1504 stammende Verzeichnis erweckt indes einen vollkommeneren Eindruck als die Schilderung von Myconius. Infolge der Überlassung der Kapelle gelangten an die Augustiner ein großes silbernes Kreuz, ein vergoldetes silbernes Kreuz mit einem Sockel, zwei Kelche, ein Pacifikal, elf Kaseln, drei Ornate, vier Chorröcke, ein Alba, mehrere Paramente und Tücher für Altäre und Kreuze, einige Leuchter, Kerzen, Sprengkessel und Ampullen, zwei Missale sowie jeweils ein Psalter und Canon für die Messfeier. ${ }^{16}$

\section{Das Augustiner-Eremiten-Kloster als Burse der Lencorea?}

Im November 1504 verpflichtete sich der Augustinerorden als Gegenleistung für die Stiftung des Klosters und dessen Ausstattung mit der Kapelle zum Heiligen Geist, Vigilien und Seelenmessen für die Vorfahren des Kurfürsten und zukünftig an seinem Todestag bzw. an dem seines Bruders Johann Jahrgedächtnisse abzuhalten. ${ }^{17} \mathrm{Im}$ selben Diplom sicherten die Augustiner dem Kurfürsten zu, geeignete Brüder zur Absicherung des universitären Lehrbetriebs zur Verfügung zu stellen. Fortan hatten tatsächlich auch stets zwei Augustinermönche Lehrstühle an der theologischen bzw. artistischen Fakultät inne. Das bekannteste Beispiel ist Martin Luther selbst, der erst den Lehrstuhl für aristotelische Ethik und sodann jenen für Theologie bekleidete. Die enge Verbindung zwischen Universität und Kloster beschränkte sich freilich nicht nur auf die Lehre seitens der Ordensgeistlichkeit, sondern nicht zuletzt auf das Studium und das alltägliche Universitätsleben.

Das Wittenberger Augustiner-Eremitenkloster diente faktisch als Burse für einen Teil der Studenten - zumindest für jene, die dem Orden angehörten und die von ihren Heimatklöstern aus allen Teilen des Reiches zum Studium an die Leucorea gesandt worden sind. Im Durchschnitt der Jahre von 1502 bis 1522 entfielen auf jedes Semester vier neu hinzugekommene Brüder, die in etwa zwischen zwei und vier Jahre in Wittenberg verblieben, sodass mit einem dauernden Bestand von etwa 15 bis 20 Mönchen auswärtiger Konvente im Augustinerkloster gerechnet

15 Zitiert nach: Sснмітт, Baugeschichte des Augustiner-Eremitenklosters (wie Anm. 2), S. 178.

16 HStA Weimar, EGA, Reg. Kk 1425, fol. $3^{\text {r }}-4^{r}$.

17 Walter Friedensburg (Hg.), Urkundenbuch der Universität Wittenberg, Teil 1 (1502-1611) (Geschichtsquellen der Provinz Sachsen und des Freistaates Anhalt, Neue Reihe 3), Magdeburg 1926, S. 12 f. (Nr. 14). 
werden muss. ${ }^{18}$ Die Heimatklöster mussten zur Unterhaltung der zum Studium geschickten Brüder einen einmaligen Betrag von acht Gulden pro Kopf entrichten. ${ }^{19}$ Zwar konnte ein Zuschuss nach einigen Semestern gefordert werden, aber insgesamt war dies zu wenig, um die anfallenden Kosten halbwegs zu decken - vor allem dann, wenn einzelne Mönche länger als vier Semester an der Leucorea studierten.

Die in diesem Beitrag zu erörternde wirtschaftliche Ausstattung des Klosters ist selbstverständlich von den sozialen Verhältnissen mitbestimmt worden. Daher die wenigen Anmerkungen zur Stärke des Konvents. Über die bedrängte Situation informiert ein Brief Luthers vom 30. Juni 1516 an den Prior des Erfurter Augustinerklosters Johannes Lang. Anlass des Schreibens ist u. a. die wirtschaftliche Situation in Wittenberg, denn Luther beschwerte sich bei ihm, dass dieser zur Entlastung seines Konvents einen Bruder nach Wittenberg abschob, obgleich doch das Eremitenkloster an der Elbe weitaus ärmer sei als das in Erfurt. Überdies - so Luther - drohe im Winter Überfüllung. ${ }^{20}$ Am 26. Oktober schrieb er abermals an Lang und teilte ihm mit, dass inzwischen 41 Personen im Kloster lebten, ${ }^{21}$ wovon mindestens die Hälfte der Professen durchweg zu den studierenden Gästen gehört haben wird.

Ein letztes Beispiel für die enge Verbindung zwischen Kloster und Universität wird durch den Schutzpatron der Leucorea, den hl. Augustin, symbolisiert, dessen auf den 28. August fallendes Jahrfest bei den Augustinermönchen feierlich begangen wurde. ${ }^{22}$ Das nahe Verhältnis beförderte eine religiöse, geistig-kulturelle und letztlich auch soziale Symbiose, sodass es auch zu wirtschaftlichen Beziehungen - freilich jenseits des Stiftungsgutes von Augustinerkloster, Allerheiligenstift und Universität, welches jederzeit streng getrennt blieb - kam. Ausdruck der engen Kooperation war die Zuweisung von Klosterräumlichkeiten an den Drucker Johann Rhau-Grunenberg für die Einrichtung seiner Offizin. ${ }^{23}$ Wie lange RhauGrunenberg im Kloster untergebracht war, ist unbekannt.

Bünger/Wentz, Bistum Brandenburg (wie Anm. 1), S. 446 f.

HStA Weimar, EGA, Reg. Kk 1434, fol. $7 \mathrm{r}$ f.

WA Briefwechsel, 1. Bd., S. 48, Nr. 18 (30. Juni 1516).

21 WA Briefwechsel, 1. Bd., S. 73, Nr. 28 (26. Oktober 1516).

22 Bünger/Wentz, Bistum Brandenburg (wie Anm. 1), S. 446.

23 Johann Friedrich Köhler, Fragmente zur Geschichte der Wittenbergischen Klöster, in: Magazin der Sächsischen Geschichte 5 (1788), S. 499-512, hier S. 511; ferner: Отто Clemen, Die lutherische Reformation und der Buchdruck (Schriften des Vereins für Reformationsgeschichte 167), Leipzig 1939, S. 19 f.; AndreAs Gössner, Die Anfänge des Buchdrucks für universitäre Zwecke am Beispiel Wittenbergs, in: Enno Bünz (Hg.), Bücher, Drucker, Bibliotheken in Mitteldeutschland. Neue Forschungen zur Kommunikations- und Mediengeschichte um 1500 (Schriften zur sächsischen Geschichte und Volkskunde 15), Leipzig 2006, S. 133-152, hier S. 145-147. 


\section{Grundversorgung des Wittenberger Konvents - die ersten Jabre}

Die anfängliche Grundversorgung des Konvents sowie auch der Universität ist aus dem Amt Wittenberg erfolgt. 1503/04 verkaufte der Kornvogt 296 Scheffel Roggen an die Universität und 139 Scheffel an die Augustiner; der Scheffel kostete vier Groschen. ${ }^{24}$ Es ist mühselig, darüber zu spekulieren, auf welche Weise die Augustiner das Getreide bezahlt haben, standen sie doch wirtschaftlich völlig am Anfang. Allerdings ist zu bedenken, dass all jene, die ins Kloster eintraten, ihren Besitz mitbringen mussten. Indessen sind nach Wittenberg nicht wenige Brüder aus anderen Konventen übergesiedelt, und von denen, wo anzunehmen ist, sie haben die Profess in Wittenberg abgelegt, wissen wir nicht, wie umfangreich ihre Beisteuer war. Dass dies nicht gering sein konnte, dokumentiert der ehemalige Prior des Augustiner-Eremiten-Klosters in Neustadt an der Orla, der 1523 in einem Schreiben darauf verwies, er habe bei seinem Eintritt 120 Gulden eingebracht. ${ }^{25}$ Inwieweit ein solcher Grundstock eingebrachten Kapitals in Wittenberg nach 1503 genutzt werden konnte, ist völlig offen.

Über die Fortschritte bzw. Schwierigkeiten beim Bau des Augustinerklosters informieren die Wittenberger Stadtkassenrechnungen. Sie illustrieren, welche Mengen an Kalk, Mauer- und Dachsteinen angefordert und verbaut worden sind. ${ }^{26}$ Bedeutsam ist freilich der Hinweis, wie die Augustiner ihren Zahlungsverpflichtungen nachgekommen sind: Nur teilweise und zögerlich oder gar nicht, was ein Indiz für die schwierigen finanziellen Verhältnisse vor Ort ist. Allerdings half Friedrich der Weise stets aus. Für die erste Bauphase hatte der Kurfürst $400 \mathrm{fl}$. zugesichert, die aus der Kasse des Amtes Wittenberg gezahlt wurden. In einem undatierten Schreiben des Johann von Staupitz an den Kurfürsten - wahrscheinlich aus dem Jahr 1505 oder 1506 - werden 400 fl. ausdrücklich erwähnt. ${ }^{27}$ Instruktiv sind auch die Zahlen des aus Bayern stammenden Hans Herzheimer, der im Jahr 1519 Wittenberg besucht hat. ${ }^{28}$ Er scheint über den Kurfürsten durch seinen

24 HStA Weimar, EGA, Reg. Bb 2742, fol. 130 r.

25 Enno Bünz, Martin Luthers Orden in Neustadt an der Orla. Das Kloster der Augustiner-Eremiten und seine Mönche (Beiträge zur Geschichte und Stadtkultur 13), Jena 2007, S. 95.

26 Schmitt, Baugeschichte des Augustiner-Eremitenklosters (wie Anm. 2), S. 182; BüNGER/WENTZ, Bistum Brandenburg (wie Anm. 1), S. 458.

27 Johann Staupitz an Kurfürst Friedrich III. von Sachsen: Deme arme, new angefangen closter zu güte und erleibung. In welche wir vorgangen sommer wol virhundert gulden vorbowen, der summa die uns von E. f. g. zugeschickt, und noch nicht surde vil volendet. ... Ruff nur alsz der nicht weyter mag endtlich zu e. f. g. umb hulff und radt, möchte euer fürstlich gnade Gote und Sancto Augustino zu ebren und dinste etzlicher zigelsteyne zu euer fürstlich gnaden aygen baw geraten uns do mit begnaden und sunst alß unser allergnedigst herr helfen. Vgl. HStA Weimar, EGA, Reg. Kk 1427, fol. $1^{\mathrm{r}}$.

28 EnNo BünZ, Wittenberg 1519: Was ein Reisender von der Stadt wahrgenommen hat, und was nicht. Mit einer Teiledition der Aufzeichnungen Hans Herzheimers, in: HeINER LÜCK u. a. (Hg.), Das ernestinische Wittenberg: Stadt und Bewohner (WittenbergForschungen 2, 1-2), Petersberg 2013, Textband S. 9-24. 
Vetter Degenhard Pfeffinger recht gut über kursächsische Interna unterrichtet gewesen zu sein. Und Herzheimer berichtet nun - rückblickend aus dem Jahr 1519 -, dass der Fürst zum Bau des Augustinerklosters „etliche Tausend Gulden“ gegeben habe. ${ }^{29} \mathrm{Ob}$ seine Zahlenangabe zuverlässig ist, sei dahingestellt. Vielleicht wollte er auch nur ausdrücken, dass Friedrich der Weise das Augustinerkloster und den Konvent insgesamt stark gefördert hat. Von 1502 bis 1519 dürften es wahrscheinlich tatsächlich etliche Tausend Gulden gewesen sein.

Wie sind nun solche Summen einzuordnen? Wie weit konnte mit einem dreioder vierstelligen Betrag ein respektabler Bau vorangetrieben werden? Aus kurfürstlicher Perspektive waren einige Hundert Gulden relativ wenig, ließen doch die sächsischen Kurfürsten seit Beginn der 1470er-Jahre alljährlich für viele Tausend Gulden ihre Burgen und Schlösser um- und ausbauen. Beispielsweise gab Kurfürst Ernst im Durchschnitt der sieben Rechnungsjahre von 1477/78 bis 1483/84 jährlich rund 3.800 fl. für den Schlossbau aus. ${ }^{30}$ Infolge der Regierungsübernahme durch Kurfürst Friedrich III. 1486 änderte sich quantitativ daran grundsätzlich nichts. ${ }^{31}$ Insofern erscheinen die von Staupitz erwähnten 400 Gulden im Vergleich zum fürstlichen Bauprogramm als wenig. Allerdings ist zu bedenken, dass dieser Betrag auch ausreichend gewesen ist, um zumindest den Bau im Grund voranzutreiben. Da es nicht unproblematisch ist, den Schlossbau der Fürsten als Vergleich heranzuziehen, seien alternative Beispiele genannt. Der Bau des stattlichen und repräsentativen Melanchthon-Hauses im Jahre 1536 verschlang knapp 850 Gulden, wovon übrigens der Kurfürst 400 fl. und die Universität 200 fl. beigesteuert haben. Wenige Jahre zuvor, im Jahre 1530, beabsichtigte Kurfürst Johann, die Befestigung der Stadt zu erweitern. Zu dem Zweck sollten 30 Häuser im Coswig-Viertel abgerissen werden, deren Wert auf insgesamt $6.900 \mathrm{fl}$. geschätzt wurde. Für den Bau neuer Häuser waren 4.000 fl. vorgesehen. ${ }^{32}$ Zwar konnte der Abbruch aufgrund des Einspruchs der Bürger, der Stadt sowie nicht zuletzt Martin Luthers verhindert werden, aber die finanzielle Kalkulation zeigt, welche Summen selbst der Bau schlichter Gebäude verschlingen konnte.

29 Von dem Augustiner closter. Dar zue habn ir churf(urstlich) gn(aden) in obermelte statt ain schönes closster Augustiner ordens Cistercensis von newem auf gericht vnd zw dem paw ettlich tausent gulden gegebenn. In gemeltem closster wonedt der hochgelert ond geistlich beruembt theologus doctor Martinus Lutter, ain vber treffenlich gelerter predicator, welicher in seinen predigen in weitten landen wol beruft ist, ond gar vil schoner leer inn druck gepracht ond aus geen lassen. Er list auf der schuell täglich 1 lection vnd dar zue ain predig in der pfar am feiertag. Vgl. Bünz, Wittenberg 1519 (wie Anm. 28), S. 22 f.

30 Hugo Grosse, Die kursächsischen Finanzen am Ausgang des Mittelalters, Phil. Diss. Leipzig 1914, S. 187.

31 Uwe Schirmer, Kursächsische Staatsfinanzen (1456-1656). Strukturen - Verfassung Funktionseliten (Quellen und Forschungen zur sächsischen Geschichte 28), Stuttgart 2006, S. 302-322.

32 Manfred Straube, Wittenberg in den Anfangsjahren der Universität und der Reformation. Wirtschaftliche Herausforderungen und soziale Probleme am Beginn einer neuen Stadtentwicklung, in: Stefan Oehmig (Hg.), 700 Jahre Wittenberg. Stadt - Universität - Reformation, Weimar 1995, S. 431-448, hier S. 437. 
Ein letztes Beispiel soll noch einmal jene $400 \mathrm{fl}$. einzuordnen helfen. Friedrich der Weise hat Zeit seines Lebens gestiftet, geschenkt und Almosen gegeben. Aufgrund der komplexen Quellenlage wird man wohl nie exakt ermitteln können, wie viele Tausende Gulden er für fromme Zwecke ausgab - seine Wallfahrt ins Heilige Land und die Sammlung seiner Reliquien nicht mit gerechnet. Georg Buchwald unterzog sich vor vielen Jahren der Mühe, wichtige Quellen - jedoch bei weitem nicht alle - bezüglich der Frömmigkeit des Kurfürsten für die Jahre von 1487 bis 1522 durchzusehen. ${ }^{33}$ Das Gros der Spenden setzt sich aus Pfennig- und Groschenbeträgen zusammen, die Kurfürst Friedrich und sein Bruder Johann an Geistliche, Schulmeister und Chorschüler oder an Wallfahrer und Bedürftige gegeben haben. Sehr oft sind für Stifter, Klöster, Kirchen und Kapellen Wachs und Kerzen gekauft sowie vor allem auch liturgische Geräte bezahlt und herangeschafft worden.

Auch Baumaßnahmen wurden gefördert. Auf Details ist an dieser Stelle zu verzichten. $\mathrm{Zu}$ erwähnen ist jedoch, dass das Wittenberger Allerheiligenstift, das dortige Franziskanerkloster sowie eben auch der Augustiner-Eremitenorden stets unter den Empfängern von Geld- oder Getreidespenden nachzuweisen sind. Untersuchte man das Schenken und Spenden des Fürsten allein aus quantitativer Perspektive, dann ragen jene $400 \mathrm{fl}$. heraus, welche die Augustiner bekommen haben. Die Auszahlung der von Staupitz erwähnten 400 fl. lässt sich zumindest teilweise belegen. Das Geld zur Förderung des Klosterbaus ist aus dem Amt Wittenberg auf Befehl des Kurfürsten in einheimischer Silberwährung in den Jahren 1503 und 1504 ausgezahlt worden: $70 \beta$ uff schrifft m. g. h. den angustinern monchen, ytzliche quatember $50 \mathrm{fl}$. gegeben. ${ }^{34} 70$ Schock Groschen waren 4.200 Silbergroschen bzw. 200 (Rechen)-Gulden, den Gulden zu 21 Groschen gerechnet. Somit wurden im Rechnungsjahr 1503/04 insgesamt $200 \mathrm{fl}$. quartalsweise zu jeweils 50 Gulden den Augustinern gereicht. Die finanzielle Unterstützung setzte sich 1504/05 fort. Allerdings zahlte der Wittenberger Amtsschösser Anthonius Niemegk den Augustinern in jenem Jahr nur 25 fl. pro Quatember aus. ${ }^{35}$ Warum die Zahlung verringert wurde, ist nicht in Erfahrung zu bringen. Wahrscheinlich waren die Bautätigkeiten am Kloster ins Stocken geraten, denn Wittenberg glich in jenen Jahren einer wirklichen Großbaustelle, auf der zeitweise Arbeitskräftemangel herrschte. Jahre zuvor, im September 1489, schrieb der Schösser nach Kemberg bzw. Schmiedeberg und bat die dortigen Ratsleute um Maurer und Tagelöhner, da er für den Bau an Schloss und Allerheiligenstift, Brücke und Stadtgraben niemanden bekommen könne. ${ }^{36}$ Im April 1492 forderte der Kurfürst die Städte Zwickau,

33 Georg Buchwald, Zur mittelalterlichen Frömmigkeit am kursächsischen Hofe kurz vor der Reformation, in: Archiv für Reformationsgeschichte 27 (1930), S. 62-110. HStA Weimar, EGA, Reg. Bb 2742, fol. $52^{\mathrm{v}}$.

35 HStA Weimar, EGA, Reg. Bb 2744, fol. 52r .

$361 \mathrm{gr} 3 \mathrm{~d}$ ken Kemberg und Smideberg, also man anschriep, dasz sie erbeiter her schicken sulden, do man der nicht bekommen konde, die woche post Egidii. HStA Weimar, EGA, Reg. Bb 2729, fol. 63r. 
Altenburg, Jena und Pößneck auf, Maurer für den Schlossbau zu schicken, um ihn schneller voranzutreiben und $\mathrm{zu}$ vollenden. ${ }^{37}$ Damit waren freilich nicht jene Steinmetze gemeint, die aus Trier und Worms gekommen waren, um am Bau von Stift und Schloss mitzuwirken. Nach 1503 war die Situation ähnlich. Nicht zuletzt dokumentieren dies die Amtsrechnungen, denn der Schösser half finanziell nicht nur Augustinern oder Franziskanern, ${ }^{38}$ sondern er unterstützte auch einzelne Lehrkräfte oder den Bau an Universitätsgebäuden. ${ }^{39}$

Daneben bedachte der Kurfürst mit Zuweisungen auch die Wittenberger Franziskaner, die 1508 zu ihrem Bau 200 Gulden erhielten. Im Jahre 1509 bekamen die Zisterzienserinnen in Oberweimar $100 \mathrm{fl}$., wobei insgesamt $100 \mathrm{~B} \mathrm{gr}$ gegeben werden sollten, sowie - auf verschiedene Posten während des Jahres 1516 verteilt - das Wittenberger Allerheiligenstift, dem rund $127 \mathrm{fl}$. gereicht worden sind. Eine besondere Aufwendung, nicht zuletzt wegen des Volumens, waren knapp $117 \mathrm{fl}$., die der kursächsische Rat und Vertraute Hans Unbehauen erhielt. Er hatte im Auftrag des Fürsten bei der Firma Fischer in Nürnberg eine Grabplatte aus Messing für die verstorbene Frau des Herzogs Wilhelm III. anfertigen lassen, die 1497 zur letzten Ruhestätte ins Kloster Reinhardsbrunn gebracht worden war. ${ }^{40}$ Beständig und beinahe regelmäßig wurden jedoch die Wittenberger Franziskaner und Augustiner unterstützt.

Der Bau des Augustiner-Eremitenklosters war ein drängendes Problem. Ein anderes war die Versorgung des Konvents. Zwar wies der Kurfürst dem Kloster grundherrschaftliche Einkünfte im Jahr 1504 zu, aber es muss offen bleiben, ob diese zum Auskommen ausreichend waren. Die bereits angeführten Klagen Luthers von 1516 beziehen sich auf die Stärke des Konvents und somit auch auf die materielle Ausstattung. Immerhin erwähnt er in dem Schreiben vom 30. Juni, dass Erfurt vermögender sei und dass zwei Wittenberger Mendikanten wegen des Baus terminieren (sunt in terminis pro collectura structarae). ${ }^{41}$ Gleichfalls war erwähnt worden, dass jedes Heimatkloster eine einmalige Aufwandsentschädigung in Höhe von acht Gulden für einen jeden entrichtete, der im Kloster unterkam, um an der Leucorea zu studieren. Die Lebensmittelpreise auf den Wittenberger Wochenmärkten waren zu Beginn des 16. Jahrhunderts noch moderat, sodass jene acht Gulden gereicht hätten, um sich genügsam für zwei Studienjahre ernähren zu können. ${ }^{42}$ Völlig unbeachtet bleiben dabei freilich die Aufwendungen für Heiz-

37 Straube, Wittenberg (wie Anm. 32), S. 436.

$381 \beta 12$ gr vor $2 \beta$ langhe breth, ye $1 \beta$ vor $36 \mathrm{gr}$, den barfüsser monchen zcu irem bawe gegeben. Vgl. HStA Weimar, EGA, Reg. Bb 2742, fol. 52v.

3910 1/2 gr vor kachelln under eyn ofen in magister Rotstocks stuben zu machen. - $14 \mathrm{gr}$ Dictus Voyte vom lectorio iuristarum bey tagk und nacht zcuberaspen und tonnichen. Vgl. HStA Weimar, EGA, Reg. Bb 2744, fol. $95^{\mathrm{r}} \mathrm{f}$.

40 Buchwald, Frömmigkeit am kursächsischen Hofe (wie Anm. 33), S. 68-71, 92.

41 WA Briefwechsel, 1. Band, S. 48, Nr. 18 (30. Juni 1516).

42 Um 1500 waren rund 80 bis 90 Groschen notwendig, um sich leidlich ernähren zu können. Die Grundernährung bestand in dem Fall pro Kopf/Jahr aus $220 \mathrm{~kg}$ Schwarzbrot, 40 kg Schweine- oder Rindfleisch, 10 kg Hering, 1501 Dünnbier, 601 fettarmer 
material oder für etwas besseres Bier oder gar Wein sowie ganz zu schweigen von Spezereien oder Erzeugnissen des Buchdrucks. Welche Kosten für einen Studenten anfielen, dokumentiert der Brief des Basler Studenten Philipp Bechius an Oswald Myconius vom Mai des Jahres 1542, wonach die jährlichen Lebenshaltungskosten zwischen 25 und 50 fl. gelegen haben. Der spätere Verleger der Schriften Georg Agricolas erwähnt, dass er allein $18 \mathrm{fl}$. jährlich für Speise und Trank ausgeben müsse. ${ }^{43}$ Vergleichbare Nachrichten liegen aus den zwanziger Jahren vor. So hat sich Caspar Tryller, Vikar an der Saalfelder Stadtkirche, 1523 mit 90 Gulden von seinem Lehn abfinden lassen, um auf diese Weise sein Studium in Wittenberg finanzieren zu können. Drei Jahre hat er an der Leucorea studiert. ${ }^{44}$

Selbst wenn die kräftigen Preissteigerungen seit Beginn der dreißiger Jahre in Betracht gezogen werden, wird deutlich, mit welchen alltäglichen Problemen die Studenten und folglich auch der Konvent der Augustiner-Eremiten zu kämpfen hatten. Um diese These untermauern zu können, sei kurz vorausgegriffen. Das Wittenberger Augustiner-Eremitenkloster ist 1503 vom Kurfürsten fundiert worden. Er wies ihm Zins- und Geschosseinkünfte in Höhe von 42 Schock und 31 Groschen zu (2.551 Groschen). Setzt man voraus, dass eine erwachsene Person um das Jahr 1500 jährlich zum Leben rund vier bis fünf Gulden (84 bis 105 Groschen) an Bargeld benötigte - sofern sie völlig auf den Lebensmittelmarkt angewiesen war -, dann wird deutlich, dass der Wittenberger Konvent, der ohne die Augustiner aus den anderen Klöstern wohl 20 Brüder umfasst hat, stets sorgsam kalkulieren musste, um die Versorgung insgesamt abzusichern. Zwar standen dem Kloster auch Naturaleinkünfte zu, diese flossen hingegen nicht besonders üppig.

\section{Die gestiftete Grundherrschaft zu Dabrun (1503-1509)}

Zwar versorgten sich die Augustiner-Eremiten teilweise durch Terminieren, aber auf diese Weise konnten sich die Konvente nicht unterhalten. Die Basis des wirtschaftlichen Lebens waren Grundstücksübereignungen, Güter- oder Rentenschenkungen oder das Übertragen wiederkäuflicher Zinse. Die Grundstücke und Güter bewirtschafteten die Klöster entweder in Vorwerken selbst oder das Land

Milch und 10 kg Salz. Vgl. Uwe Schirmer, Ernährung im sächsischen Erzgebirge zu Beginn des 16. Jahrhunderts. Produktion, Handel und Verbrauch, in: Rainer Aurig u. a. (Hg.), Landesgeschichte in Sachsen. Tradition und Innovation (Studien zur Regionalgeschichte 10), Bielefeld 1997, S. 129-144, hier S. 133; Ders., Ernährung und Lebensmittelverbrauch im mitteldeutschen Raum (1470-1550), in: Luthers Lebenswelten (wie Anm. 2), S. 267-275; Manfred Straube, Handel und Verbrauch von Nahrungsmitteln im Umfeld Martin Luthers, in: ebd., S. 277-298.

43 Theodor Kolde (Hg.), Analecta Lutherana. Briefe und Actenstücke zur Geschichte Luthers. Zugleich ein Supplement zu den bisherigen Sammlungen seines Briefwechsels, Gotha 1883, S. 380-382.

44 Moritz Mitzenheim, Die erste Kirchen- und Schulvisitation in Saalfeld im Jahre 1525 (Heimatbücher der Kirchkreise Saalfeld und Gräfenthal 1), Saalfeld 1927, S. 10. 
war an grundherrliche Bauern vergeben, die dem Kloster Geld- und Naturalabgaben lieferten und Frondienste leisteten. Teilweise - wie beispielsweise die Grimmaer Augustiner - pflegten und unterhielten sie Weinberge und Karpfenteiche. Inwiefern sind dem Wittenberger Augustinerkloster regelmäßige Einkünfte zugeflossen und wann versiegten sie? Im Vergleich zu anderen Klöstern, die im ernestinischen oder albertinischen Sachsen beheimatet waren, ist die Quellenlage schlecht. ${ }^{45}$ Vor allem aufgrund der Überweisung der ursprünglichen Klostereinkünfte an den Kurfürsten im Sommer 1525 und der vorangegangenen Auflösung des Konvents sind keine Akten zu Einkünften, Ausgaben, Inventaren usw. angefertigt worden, so wie sie vergleichsweise von den Visitatoren infolge von Visitation, Sequestration und Säkularisation fast zu jedem Stift oder Kloster angelegt worden sind. ${ }^{46}$

Dem Wittenberger Augustinerkonvent standen seit Ende des Jahres 1503, spätestens jedoch seit 1504, regelmäßige grundherrliche Einnahmen zu. Kurfürst Friedrich der Weise hatte ihm die Dörfer Dabrun (südöstlich Wittenberg) und Kleinzerbst (nordöstlich Schmiedeberg) sowie die Wüstung Rötzsch übereignet. Lehnbriefe des späten 15. Jahrhunderts sowie ein Zinsregister von 1503 dokumentieren, dass dies eine kleine, aber nicht völlig unrentable Grundherrschaft mit Sitz in Dabrun war. Mit Dabrun sowie den dazugehörigen Ober- und Niedergerichten war der Bruder des Heinrich von Kanitz, Dietrich von Kanitz, im Jahr 1473 belehnt worden. Er hatte drei Lehnpferde zu unterhalten; zudem wird erwähnt, dass auf der wüsten Mark Rötzsch eine Schäferei sei. ${ }^{47} 1476$ erlangte Sigmund von Miltitz die Anwartschaft auf Dabrun mit Sattelhof und Vorwerk.48 In der Dabruner Kirche hatte die Familie von Kanitz 1492 eine Stiftung für abzuhaltende Seelmessen eingerichtet; zur Ausstattung war von Günther Löser eine Hufe Landes gekauft und der Kirche übertragen worden.49 Die Pfarre war 1503 ebenfalls dem Augustinerkloster unterstellt worden. Nach dem Verkauf der Grundherrschaft seitens des Klosters an Günther von Staupitz verblieb das Patronat beim Kloster. Ausdrücklich bestätigt dies eine Beschwerde des Konvents beim Kurfürsten aus dem Jahr 1520, in der die Mönche über das unbefugte Einschlagen von Eichenholz

45 Seidemann, Luthers Grundbesitz (wie Anm. 8), S. 480.

46 Vgl. die Zusammenschau bei: Alfred Hilpert, Die Sequestration der geistlichen Güter in den kursächsischen Landkreisen Meißen, Vogtland und Sachsen 1531 bis 1543, in: Mitteilungen des Altertumsvereins zu Plauen 22, 1912, S. 1-136.

47 HStA Weimar, Copial B 6, fol. 105 f. - Testamentsvollstrecker des Heinrich von Kanitz war der Generalvikar Johann von Staupitz. Vgl. BüNGER/WENTZ, Bistum Brandenburg (wie Anm. 1), S. 456 f.

48 HStA Weimar, Copial B 6, fol. 130v.

49 BüngeR/Wentz, Bistum Brandenburg (wie Anm. 1), S. 456 f. Das Zinsregister für Dabrun von 1503 nennt namentlich einen Kossäten, der under dem gotzhausze sytzt. HStA Weimar, EGA, Reg. Bb 98, fol. 21v. 
auf dem Pfarrlehn durch Staupitz klagen. ${ }^{50}$ Nach des Klosters Auflösung ging das Patronat an den Kurfürsten über. ${ }^{51}$

Zwischen 1492 und 1503 verstarb Heinrich von Kanitz. Nach dessen Tod zog Kurfürst Friedrich das Lehn ein; die Anwartschaft des Sigmund von Miltitz blieb unwirksam. Das erwähnte Wittenberger Amtszinsregister weist zum Jahr 1503 für beide Dörfer Erträge von insgesamt 40 Schock 55 Groschen aus. Die Einnahmen verteilen sich wie folgt.

Dabrun: Dis nachgeschrieben zcynse sint von Heynrich Kanytz an mey. gnedygst hern komen. Dorf Dabrun. Item Richter $3 \beta 40 \mathrm{gr}$ vom kruge und seynen guthern, das geboren sich $2 \beta$ vom schenken, daron $m$. g. h. das lyehen. Item $H$ ans Liebe $3 \beta$ von seyne guthe und 3 hufen. Item Galle Krewsel $4 \beta$ von 4 buffen. Item Kerten Ryetz $4 \beta$ von 4 huffen. Item Peter Heynrich $3 \beta$ von drey buffen, ist dienst frey und 1 lebenman, helt 1 lehnpferdt. Item Paul Steynkopf $3 \beta$ von 2 buffen. Item Dict. Heynrich $4 \beta$ von 4 buffen. Item Matt. Brunstorff $2 \beta$ von 2 buffen. // Item Hans Rape $3 \beta 36$ gr von $31 / 2$ buffen und 1 wüste hoffe. Item Merten Meyfrid $1 \beta 42 \mathrm{gr}$ von 3 huffen. Item Lawbe $10 \mathrm{gr}$. Item Lodeweyg Mersn. [?] $10 \mathrm{gr}$. Item Mewis Bünne $14 \mathrm{gr}$. Item Peter Brosze $15 \mathrm{gr}$. Item Reycharth $8 \mathrm{gr}$. Item Brosze Richter $10 \mathrm{gr}$. Item Mewis Fischer und Jorge Voyth dynen und zcynsen kein gelth. Summa $33 \beta 5$ gr.52 In Dabrun stand ein Dorfkrug, in dem der Dorfrichter das Schankrecht besaß. Das Lehen lag beim Kurfürsten. Wahrscheinlich werden dazu noch ein bis zwei Hufen Landes gehört haben. Ausgewiesen ist der Besitz von $28 \frac{1}{2}$ Hufen, die neun Bauern bewirtschafteten. Der relativ hohe Hufenbesitz darf nicht über die allgemeinen Verhältnisse hinwegtäuschen, denn das Dorf lag in der hochwassergefährdeten Niederung der Elbe. ${ }^{33}$ Unterhalb der bäuerlichen Schicht lebten noch sechs Kossäten und zwei fast völlig Mittellose im Dorf. Insgesamt zinst die Gemeinde drei Schock und fünf Groschen. Bemerkenswert an diesem Dorf ist neben dem Dorfkrug (Erbgericht) noch der Bauer Peter Heynrich, der dienstfrei war und ein Lehnpferd halten musste. Er besaß den Sattelhof, den einst Heinrich von Kanitz bewohnt hatte. Im Lehnbrief von 1473 waren indes drei Lehnpferde genannt. Anstatt der drei Lehnpferde wurde 1503 nur noch ein Pferd gehalten, denn der Dorfrichter bezahlte anstelle der zwei Lehnpferde nunmehr zwei Schock Groschen. Eine dingliche Leistung war in einen Geldbetrag umgewandelt worden. Diese Leistung zählte als grundherrlicher Ertrag und stand dem Grundherrn zu. Gericht, Lehnpferd, Sattelhof und Vorwerk weisen zweifelsfrei auf den einstigen Herrschaftssitz hin.

50 HStA Weimar, EGA, Reg. Kk 1431.

51 Pallas, Registraturen der Kirchenvisitationen (wie Anm. 14), S. 99.

52 HStA Weimar, EGA, Reg. Bb 98, fol. $16^{\mathrm{r}} \mathrm{f}$.

53 Friedrich Kalle, Beispiele für Veränderung der Flußläufe in geschichtlicher Zeit (Kartenblatt 7), in: Otto Schlüter/Oskar August (Hg.), Atlas des Saale- und mittleren Elbegebietes, Leipzig ${ }^{2} 1958$. 
Völlig anders war hingegen Kleinzerbst strukturiert: Dorff Zcerwischnn. Item gemeyn $1 \beta$ vom fybe. Item $1 \beta$ von huffen. Item $40 \mathrm{gr}$ von der lanthweyde. Item $40 \mathrm{gr}$ von der muldicken zcyns. Item $40 \mathrm{gr}$ weyde gelt. Item $16 \mathrm{gr}$ trifft gelth. Item $40 \mathrm{gr}$ wecht [er] gelth. Item $25 \mathrm{gr}$ walkwegelth [?]. Item $20 \mathrm{gr}$ hamel und kalp gelt. Item $9 \mathrm{gr}$ garbengelt. Item der Richter von eyn brethe (sc. Breite; ein Stück Land). Item Urban Osterwitz $15 \mathrm{grvon} 1$ breythe. Item Valten Richter $15 \mathrm{gr}$ von 1 breythe. Item $15 \mathrm{gr}$ Jacoff Korwyn, $14 \mathrm{gr}$ (davon von) 1 breythe. Item Bartel Kyche $10 \mathrm{grvon} 1$ breythe. Item Richter zcu Meßwitz (sc. Merschwitz) $30 \mathrm{gr}$ von 1 breythen. Item Michel Jeske $4 \mathrm{gr}$ von 1 breythen. Summa $7 \beta 50 \mathrm{gr} .{ }^{54}$ Bei aller gebotenen Vorsicht hat es den Anschein, dass das Dorf durch unterbäuerliche Schichten (Kossäten) geprägt war, was sich nicht zuletzt in der Gewichtung des Zinsertrages zwischen beiden Dörfern niederschlägt. Trotzdem - beide Dörfer betrachtet waren jene 40 B 55 gr (knapp 117 fl.) eine beachtliche Summe. Neben dem Erbzins hatte aus Dabrun jeder Bauer sechs Groschen und jeder Kossäte zwei Groschen Bete bzw. Geschoss zu zahlen. ${ }^{55}$ Es waren nochmals 1 \& 36 gr, sodass sich die gesamten Bargeldeinnahmen des Klosters auf 42 ß 31 gr bzw. 121 fl. 10 gr beliefen. Hinzu kamen noch Naturalabgaben, über die ebenfalls detailliert das Zinsregister von 1503 informiert. Aus Dabrun waren es 15 Scheffel Hafer, sechs Gänse und 21 Hühner; die Bauern von Kleinzerbst zinsten jeweils neun Scheffel Roggen und Gerste sowie 91 Hühner. ${ }^{56}$

Die Augustiner bekamen vom Kurfürsten nicht nur die beiden Dörfer übertragen, sondern auch das Vorwerk in Dabrun mit dazugehörigem Vieh. Dazu weist die Amtsrechnung von 1503/04 aus: 92 Rinder, 25 Schweine, 451 Schafe, 90 Hühner und neun Gänse. ${ }^{57}$ Hinzu kamen etliche Scheffel Korn, Gerste, Hafer, Erbsen und Malz, wobei jedoch nicht immer deutlich wird, ob diese zum Vorwerk gehörten oder ob es eine vom Kurfürsten befohlene Zuwendung war. Bemerkenswert ist zumindest die Notiz des Wittenberger Schössers, dass es sich bei Gerste und Hafer um Saatgetreide gehandelt hat. ${ }^{58}$ Mit anderen Worten: Der Kurfürst und die Wittenberger Amtsverwaltung nahmen an, dass die Augustiner den Vorwerksbetrieb in Dabrun aufrechterhielten. Auf welche Weise das Vorwerk bewirtschaftet wurde, wissen wir nicht. Der Vergleich mit den Grimmaer Augustinern zeigt indes, dass solche Vorwerke von Tagelöhnern und frondienstpflichtigen Bauern bewirtschaftet worden sind. ${ }^{59}$

54 HStA Weimar, EGA, Reg. Bb 98, fol. 17r.

55 HStA Weimar, EGA, Reg. Bb 98, fol. 21 v; Kleinzerbst hatte jedoch kein Geschoss zu entrichten!

56 HStA Weimar, EGA, Reg. Bb 98, fol. 29v $35^{\mathrm{v}}, 38^{\mathrm{v}}$.

57 HStA Weimar, EGA, Reg. Bb 2742, fol. $135^{\mathrm{r}}, 138^{\mathrm{v}}, 147^{\mathrm{r}}-149^{\mathrm{r}}$.

58 Ausgabe gerste: 90 sch gersten den augustinern zcum samen zcu Dabrun geantwort. HStA Weimar, EGA, Reg. Bb 2742, fol. 131'; Ausgabe hafer: 100 sch den augustinern zcum samen mit dem vorwerge Dabrun überantwortet. Ebd., fol. $133^{\mathrm{v}}$.

59 Codex diplomaticus Saxoniae regiae, II. Hauptteil, Bd. 15: Urkundenbuch der Stadt Grimma und des Klosters Nimbschen, hrsg. von Ludwig Schmitt (im Folgenden: CDS II/15), Leipzig 1895, S. 151-154 (Nr. 233). 
Der Wittenberger Konvent wird mit den baren und naturalen Zinserträgen leidlich ausgekommen sein. Es erscheint als sicher, dass das grundherrliche Einkommen komplett für die tägliche Hauswirtschaft in Küche und Keller verbraucht wurde. Mit anderen Worten: Der Klosterbau konnte damit nicht finanziert werden, ebenso wenig der Aufbau einer Bibliothek; dazu reichten die Einnahmen nicht aus. Es wird den Augustinern einzig möglich gewesen sein, Bauholz zu schlagen. Das unberechtigte Abholzen der Eichen durch Günther von Staupitz 1520 ist dafür ein Indiz. Die Grundherrschaft warf Gewinn ab; das Vorwerk in Dabrun war gut ausgestattet worden. Die Erträge aus der Viehwirtschaft werden die Versorgung des Konvents mit abgesichert haben. Der Kurfürst hatte somit alles getan, um die Grundversorgung des Konvents zu gewährleisten. Die Fundation war eine Art Mischfinanzierung: Die Geldeinkünfte wurden durch geringe grundherrliche Naturaleinkünfte ergänzt. Das Gros der Naturaleinkünfte kam indes aus dem Vorwerk Dabrun. Den Bau und die Ausstattung des Klosters, dies wird dem Kurfürsten Friedrich, Johann von Staupitz und dem Landrentmeister Degenhart Pfeffinger bewusst gewesen sein, konnten die Mönche aus eigner Kraft nicht finanzieren. Aus diesem Grund stellte der Fürst eine erste Anschubfinanzierung in Höhe von 400 fl. bereit; nicht zuletzt aus diesem Grund bat Staupitz den Landesherrn geraume Zeit später, den Bau weiter finanziell zu unterstützen. Auf alle Fälle war das vom Kurfürsten in den Jahren um 1503/04 Gestiftete und Übertragene ausreichend, um eine gedeihliche Entwicklung des Wittenberger Augustiner-Konvents absichern zu helfen.

\section{Rentenkauf}

Der Wittenberger Konvent hat im Januar 1509 Dabrun und Kleinzerbst mit Sattelhof und Vorwerk sowie allen grundherrlichen Rechten und Einkünften an Günther von Staupitz abgetreten. Staupitz war der Bruder des Generalvikars der Observanten-Kongregation. Der Wert der gesamten Grundherrschaft wurde mit 2.400 fl. angeschlagen. Konkret: Die Nutzung des gesamten Objektes stand allein Günther von Staupitz zu. Selbstverständlich hatte er den Mönchen keinen „müden Heller“ bar ausgehändigt. Ihm stand allein das Nutzungsrecht zu, welches mit sechs Prozent verzinst wurde. Tatsächlich zahlte er vorerst regelmäßig einen Jahreszins in Höhe von $144 \mathrm{fl}$. an das Kloster. Das Geld hatte er zum Weihnachtsfest in Wittenberg zu hinterlegen. Das Geschäft stand unter dem Vorbehalt des Rückkaufs bei halbjährlicher Kündigung. 60 Über die Ursachen des Rentenkaufs seitens der Augustiner kann nur spekuliert werden. Einerseits ist an die Hochwassergefahr zu denken, die stets viele Bauern in der Elbniederung an den Rand des Ruins brachte. ${ }^{61}$ Andererseits erforderte die Führung einer Grundherr-

60 HStA Weimar, EGA, Urkunde, Nr. 4534 (1509 Januar 16).

61 Uwe Schirmer, Alltag, Armut und soziale Not in der ländlichen Gesellschaft - Beobachtungen aus dem kursächsischen Amt Wittenberg (1485-1547), in: Stefan Oehmig 
schaft und eines Vorwerks Geschick und Präsenz. Der Prior hätte einen Verwalter vor Ort einsetzen müssen, der die Arbeiten organisierte. Offensichtlich ist dies, aus welchen Gründen auch immer, nicht geschehen, sodass es zum Verkauf kam. Nach wenigen Jahren kam Staupitz seinen Zahlungen nicht mehr nach. In einem undatierten Schreiben an den Kurfürsten klagen die Augustiner über ausbleibenden Zins sowie über nicht zurückerstattetes Getreide und schuldigen Kalk. Das Getreide und den Kalk hätten sie ihm bei der Übergabe geborgt. Den Kalk hätte er u. a. zum Weißen des Sattelhofes benutzt. ${ }^{62}$ Wahrscheinlich stammt das Schreiben nicht aus den ersten Tagen der beginnenden Reformation. Vielleicht hat Staupitz Gründe namhaft machen können, warum er in Verzug geraten ist. Auf alle Fälle wurde nachfolgend der Zinssatz auf fünf Prozent gesenkt, denn in den beiden erhaltenen Verzeichnissen über das Einkommen des Wittenberger Augustinerklosters aus den Jahren 1522 und 1524 erscheint er mit einem zu zahlenden Zinsbetrag in Höhe von $122 \mathrm{~B}$ und 12 gr. ${ }^{63} \mathrm{Ob}$ die Modifizierung des Rentengeschäfts mit der Ausstellung eines Lehnbriefs für Günther von Staupitz im Jahre $1518 \mathrm{zu}$ erklären ist, bleibt offen. Instruktiv ist jedoch, dass nunmehr nicht nur Dabrun, Kleinzerbst und die wüste Mark Rötzsch erwähnt werden, sondern auch die Trift auf der Wüstung Boos. ${ }^{64}$ Damit sind die Konturen des vom Kurfürsten gestifteten Grundbesitzes an die Augustiner aus dem Jahr 1503 relativ deutlich umrissen.

Anfang Mai des Jahres 1509 kauften die Augustiner vom Kleinen Chor des Allerheiligenstifts eine Hauptsumme von 400 fl., die sie mit einem Jahresertrag von $20 \mathrm{fl}$. zu verzinsen hatten; auch diese Transaktion ist nach $1522 \mathrm{zu}$ belegen. ${ }^{65}$ Bemerkenswert ist, dass die beiden Rentengeschäfte des Jahres 1509 miteinander verbunden waren. So konnten die 144 fl., die Staupitz dem Kloster zu zahlen hatte, für den Fall gesperrt bzw. ins Allerheiligenstift gewiesen werden, so die Augustiner ihren Verpflichtungen nicht nachkamen. Die Sperre sollte ab einem Zahlungsrückstand von $80 \mathrm{fl}$. (also von vier säumigen Jahresraten) in Kraft treten. ${ }^{66}$ Tatsächlich kamen die Augustiner ihren Verpflichtungen gegenüber dem Allerheiligenstift nicht nach - jedoch erst nach 1518. Staupitz nahm dies zum Vorwand, um seine Zinszahlungen an das Kloster einzustellen, sodass die Augustiner ihrerseits nach 1519 die finanziellen Pflichten gegenüber dem Stift nicht mehr erfüllten. ${ }^{67}$

Für das Wittenberger Kloster waren zudem wiederkäufliche Zinsen aus dem Amt Colditz einträglich. Diese Einkünfte hatte Günther von Staupitz zu Dabrun angewiesen. Um Stiftungen wird es sich nicht gehandelt haben; vielmehr stehen

(Hg.), Medizin und Sozialwesen in Mitteldeutschland zur Reformationszeit (Stiftung Luthergedenkstätten in Sachsen-Anhalt), Leipzig 2007, S. 115-142, hier S. 138 f.

62 HStA Weimar, EGA, Reg. Kk 1426.

63 HStA Weimar, EGA, Reg. Kk 1434, fol. $2^{\mathrm{r}-4^{\mathrm{v}}}$ (1524), fol. $6^{\mathrm{r}-7^{\mathrm{r}}}$ (1522).

64 HStA Weimar, EGA, Copial B 8, fol. $176^{\mathrm{r}}-178^{\mathrm{r}}$.

65 HStA Weimar, EGA, Reg. Kk 1434, fol. $2^{\mathrm{r}}$ f., $6^{\mathrm{v}} \mathrm{f}$.

66 BüngER/WenTZ, Bistum Brandenburg (wie Anm. 1), S. 453 f.

67 BüngER/Wentz, Bistum Brandenburg (wie Anm. 1), S. 454. 
diese Renten in Verbindung mit dem Verkauf der Grundherrschaft Dabrun. Jedenfalls hatten im Juli 1514 Christoph und Wolf von Brösen den Augustinern Einkünfte von $90 \mathrm{fl}$. aus den Dörfern Motterwitz und Förstgen sowie den Vorwerken zu Ostrau und Dürrweitzschen verschrieben. ${ }^{68}$ Diese Rente wurde 1522 ebenfalls als finanzieller Ertrag des Kloster aufgezeichnet: $90 \mathrm{fl}$. von Christoff von Bresen von dem dorf Motterwitz und Muschaw uf Nativitatis Christi, von wegen Günther von Staupitz. ${ }^{69}$ Betrachtet man jedoch nur den Gunstbrief des Jahres 1514, dann ist weder von Günther von Staupitz noch von dem Dorf Muschau zu lesen. 70 Über die einzelnen Hintergründe ist nichts bekannt.

Ebenfalls aus dem Amt Colditz kam eine von Günther von Staupitz angewiesene Rentenschuld, die durch Appel von Arras zu Marschwitz vom Dorf und Vorwerk Polditz in Höhe von 27 fl. gezahlt werden sollte. Das Kapital belief sich auf 450 Gulden. Allerdings ist der Zinssatz zwischen 1522 und 1524 von sechs auf fünf Prozent gesenkt worden. In dem bereits mehrfach erwähnten Verzeichnis allen Einkommens der Augustiner-Eremiten aus den Jahren 1522 bzw. 1524 wurde dies bereits berücksichtigt: $221 / 2 \mathrm{fl}$. von den erben Appel von Arris auf martini, ist die heuptsumma $450 \mathrm{fl}$. Zum Jahr 1524 ist jedoch zu lesen: $27 \mathrm{fl}$. von Appel zu Arras zu Marschwitz von fhorberg und dorf Poltitz uff martini, auch von wegen Günthers von Staupitz. ${ }^{71}$ Letztlich ist eine Rente zu erwähnen, die den Augustinern seit 1511 zustand. ${ }^{72} 1522$ notierte der Wittenberger Schösser zu dieser Rente: $5 \mathrm{fl}$. von den edelleuten von Hayn und Moltzan bey der Neustadt, ins fünfte jabr binderstellig blieben und schwerlich fürbas zu überkommen..$^{73}$

Damit ist die Bestandsaufnahme hinsichtlich des Einkommens des Augustinerklosters zu Wittenberg abgeschlossen. 1522 beauftragte der kurfürstliche Rat Haubold von Einsiedel den Wittenberger Schösser Gregor Burger, die gesamten Einnahmen aufzuzeichnen. Dass sie sehr unregelmäßig bzw. nicht gezahlt worden sind, wurde erwähnt. 1522 setzten sie sich folglich aus $122 \mathrm{fl} .12 \mathrm{gr}$ von Günther von Staupitz, $90 \mathrm{fl}$. von Christoph von Bresen, $27 \mathrm{fl}$. von Appel von Arras und den

68 HStA Weimar, EGA, Copial B 8, fol. 346r .

69 HStA Weimar, EGA, Reg. Kk 1434, fol. $2^{\text {r }}$. Christoph von Brösen war der Besitzer des Gutes Motterwitz und hatte diese Rente für Günther von Staupitz zu leisten. Die Hauptsumme belief sich auf $1.500 \mathrm{fl}$.; sie wurde also mit sechs Prozent verzinst. Vgl. auch: ebd., Reg. Kk 1432.

70 Christoff und Wolffen von Bresen, gebruder, haben auf dem dorff Motterwitz, dem vorwerk zu Ostra, dem vererbten vorwerck zu Weitschen und auf dreien mennern zu Fürstgen neuntzig gulden dem prior und sambung des closters sancti Augustini zu Wittenberg vor $1.500 \mathrm{fl}$. heuptsumma auff widerkauff verkeufft. Und ist inen von unserem gn. Herrn darüber gunst mit vorbebalt der ritterdienst und ander obirkeit auff drey jar lang gegeben worden. Actum Torgaw mittwochs sand margarethen abend anno domini 1514. Vgl. HStA Weimar, EGA, Copial B 8, fol. 346r.

71 HStA Weimar, EGA, Reg. Kk 1434, fol. $2^{\mathrm{r}}, 6^{\mathrm{v}}$.

72 HStA Weimar, EGA, Copial D 6, II, fol. $173^{\mathrm{r}} \mathrm{f}$.

73 HStA Weimar, EGA, Reg. Kk 1434, fol. 6v 
5 fl. von den Edelleuten zusammen; insgesamt waren es 244 fl. 12 gr. Dem standen Ausgaben in Höhe von 58 fl. entgegen, sodass es einen Überschuss von 186 fl. gab. ${ }^{74}$ Naturaleinkünfte standen dem Kloster nicht zur Verfügung. Die Geldeinkünfte waren indessen nicht unbeträchtlich. Es sei daran erinnert, dass sie sich 1503/04 auf knapp 117 fl. belaufen haben. Infolge der Übertragung von Dabrun an Staupitz sollten jährlich sogar 144 fl. einkommen. Nunmehr, im Jahre 1522, wurde die Gesamteinnahme sogar auf über $244 \mathrm{fl}$. beziffert. Insgesamt war dies eine respektable Summe; damit konnte der Konvent versorgt und die Gebäude des Klosters einigermaßen unterhalten werden. Diese Einschätzung gründet sich auf den Vergleich der Nettoeinnahmen der kursächsischen Ämter. ${ }^{75}$ Natürlich warfen die einkommensstärksten Ämter in jenen Jahren das Fünf- bis Zehnfache und noch mehr ab; allerdings gab es einige Ämter, die mit derartigen Beträgen auskommen mussten. Mit einigen Hundert Gulden konnte freilich weder eine große Amtsburg saniert, noch ein Kloster gebaut werden. Dazu bedurfte es anderer Beträge. Dennoch: Zumindest am Vorabend der Reformation schien das Wittenberger Augustinerkloster nicht schlecht ausgestattet gewesen zu sein.

Dazu ein Vergleich: Es ist nicht unproblematisch, die Einkünfte der Stifter und Klöster des frühen 16. Jahrhunderts exakt zu vergleichen. Zu viele Unsicherheitsfaktoren, insbesondere bei den grundherrlichen Naturaleinkommen sowie bei den Erträgen aus den Vorwerkswirtschaften, behindern eine solche Gegenüberstellung - ganz zu schweigen von den Eigenarten der Stifter, Orden und Kongregationen. Allerdings ist ein Vergleich aufgrund der überlieferten Sequestrationsakten ansatzweise möglich. Er förderte bezüglich des Einkommens zwischen den Wittenberger Augustinern und den sächsischen, meißnischen und vogtländischen Stiftern und Klöstern, die Ende der 1520er- und zu Beginn der 1530er-Jahre sequestriert worden sind - bei aller gebotener Vorsicht -, eine Erkenntnis zutage: Im „Ranking“ läge das Wittenberger Augustiner-Eremiten-Kloster mit auf den hinteren Rängen. Die letzten Plätze hatten freilich die Franziskaner aus Torgau und die Zwickauer und Plauener Dominikaner inne, wo die Erträge so gering waren, dass sie bereits vor der Sequestration in die dortigen Gemeinen Kästen geschlagen worden sind. Die Wittenberger Augustiner mit ihren rund $244 \mathrm{fl}$. an baren Einkünften wären aber noch vor den Dominikanerinnen aus Weida (rund $180 \mathrm{fl}$.) zu finden. ${ }^{76}$

Doch dies kann nur zur Illustration dienen. Beispielsweise standen dem Neustädter Augustinerkonvent jährliche Bargelderträge von nur $741 \frac{1}{2} \mathrm{fl}$. zu! Allerdings waren dort die Naturaleinkünfte bedeutend umfangreicher. ${ }^{77}$ Letztlich sei erwähnt, dass die großen Stifter (St. Georgen in Altenburg, die dortigen Chorherren

74 HStA Weimar, EGA, Reg. Kk 1434, fol. ${ }^{\text {r }}$. Ausgabe. 20 fl. in den cleynen chor aller beyligen stift zu Wittenberg, $10 \mathrm{fl}$. zu joh. Bapt. Und $10 \mathrm{fl}$. nativitatis christi. $20 \mathrm{fl}$. gein Meidburg (sc. Magdeburg) den testamentar., heißen Falcken, uf ostern. 18 fl. dem licentiaten Amstorff, chathar. Virginis. Summa der ausgab: 58 fl., restat im vorrat: $186 \mathrm{fl}$.

75 Schirmer, Kursächsische Staatsfinanzen (wie Anm. 31), S. 294 f., 914 et passim.

76 Hilpert, Sequestration der geistlichen Güter (wie Anm. 46), passim.

77 BüNz, Luthers Orden in Neustadt (wie Anm. 25), S. 98. 
oder das Wittenberger Allerheiligenstift) über vierstellige Bareinnahmen verfügten.

Ein letzter Vergleich: Die finanziellen und materiellen Einnahmen sind das eine, das andere ist die Stärke der Konvente. Wie erwähnt, bestand die Wittenberger Gemeinschaft aus circa 20 Brüdern. Hinzu kamen noch 15 bis 20 Angehörige auswärtiger Konvente. Luther selbst hatte im Jahr 1516 die Personalstärke mit 41 angegeben; Ende 1521 lebten circa 30 Brüder im Kloster. ${ }^{78}$ Damit übertraf Wittenberg eindeutig andere Augustinerklöster. Zum Beispiel lebten in Grimma zu Beginn des Jahres 1522 (einschließlich derjenigen, die das Kloster verlassen hatten) 14 Mönche und 4 Laienbrüder, dazu mussten je zwei Köche und Wagenknechte sowie ein Hirte täglich mit versorgt werden. Insgesamt waren es 23 Personen; ${ }^{79}$ die Einnahmen der Grimmaer Augustiner beliefen sich hingegen auf rund $650 \mathrm{fl}$., wovon jedoch $450 \mathrm{fl}$. auf die Klostermühle entfielen. ${ }^{80}$ Dresden war 1404 bzw. 1412 mit insgesamt sechs Mönchen ausgestattet; 1539 waren es sieben Mönche und vier Laienbrüder; die Bargeldeinnahmen beliefen sich jährlich auf mindestens 270 fl. ${ }^{81} 17$ Brüder lebten im Konvent des Neustädter Augustiner-Eremitenklosters am Vorabend seiner Auflösung. ${ }^{82}$ Schließlich sei noch das Augustinerkloster Sangerhausen angeführt. Die dortige Gemeinschaft war infolge der Pest im Jahr 1519 um acht Brüder dezimiert worden. 1522 verließen drei Mönche das Kloster und hinterließen sieben Brüder und den Prior. ${ }^{83}$

\section{Zusammenfassung}

Nach alldem, was rekonstruierbar ist, bleibt festzuhalten, dass das Wittenberger Augustinerkloster von Anbeginn nicht schlecht ausgestattet war. Mit der Übertragung der Grundherrschaft in Dabrun hatte der Kurfürst den Konvent solide dotiert. Vor allem: Es war eine aus Natural- und Geldeinkünften bestehende Fundierung. Hinzu kam das Vorwerk. Es ist dargelegt worden, dass die Grundherrschaft 1509 zugunsten eines jährlichen Rentenertrages abgetreten wurde. In den folgenden Jahren kamen weitere Renten hinzu, sodass das Kloster im Jahr 1522 über Bargeldeinkünfte von rund 244 Gulden verfügte. Im regionalen Vergleich, ins-

78 Bünger/Wentz, Bistum Brandenburg (wie Anm. 1), S. 449-451.

79 CDS II/15, S. 151 (Nr. 233).

80 CDS II/15, S. 151 (Nr. 233); Hilpert, Sequestration der geistlichen Güter (wie Anm. 46), S. 47.

81 Codex diplomaticus Saxoniae regiae, II. Hauptteil, Bd. 5: Urkundenbuch der Städte Dresden und Pirna, hrsg. von Karl Friedrich von Posern-Klett, Leipzig 1875, S. 298 f. (Nr. 409 f.), S. 320 (Nr. 443).

82 BüNZ, Luthers Orden in Neustadt (wie Anm. 25), S. 115-123.

83 Georg Haubner, Das Augustiner-Eremitenkloster in Sangerhausen, in: Mitteilungen des Vereins für Geschichte von Sangerhausen und Umgebung 16/17 (2007/2008), S. 104-132, hier S. 124 (nach freundlichem Hinweis von Enno Bünz). 
besondere unter den mitteldeutschen Mendikanten, war dies eine nicht unansehnliche Summe, im Vergleich zu anderen großen Abteien und Stiften natürlich relativ wenig. Freilich benötigten die meisten geistlichen Institutionen Zeit, um Besitz in Form von Stiftungsgut zu akkumulieren. Insofern muss das Wittenberger Augustinerkloster als singulär angesehen werden, denn weder die kurze Lebensdauer, noch eine nicht nachzuweisende und wohl auch ausgebliebene Ablasskampagne haben die Ausstattung beeinflusst. Entscheidend war die faktische Integration in die Leucorea und damit verbunden der Wille des Kurfürsten Friedrich, die Wittenberger Universität nach besten Kräften zu fördern. Aus einer materiellen Perspektive heraus betrachtet, war dies stets nützlich für Kloster und Konvent. 